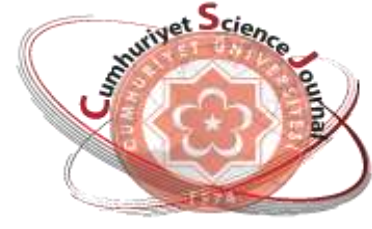

e-ISSN: $2587-246 X$

ISSN: $2587-2680$

\section{Cumburiy ot Seionee Journal \\ esy}

Cumhuriyet Sci. J., Vol.39-2(2018) 314-324

\title{
Spacelike Ruled Surfaces in Hyperbolic 3-Space
}

\author{
Tuğba MERT \\ Cumhuriyet University, Faculty of Science, Departmant of Mathematics, Sivas, TURKEY
}

Received: 25.01.2018; Accepted: 30.04.2018

http://dx.doi.org/10.17776/csj.383468

\begin{abstract}
A spacelike ruled surface in $\mathbb{H}^{3}$ is obtained by moving a spacelike geodesic along a spacelike curve. In this paper, we have studied spacelike ruled surfaces in Hyperbolic 3-space $\mathbb{H}^{3}$. We have also investigated the concepts striction point, striction curve and dispersion parameter of ruled surfaces in $\mathbb{H}^{3}$.
\end{abstract}

Keywords: Spacelike Ruled Surface, Hyperbolic Space

\section{Hiperbolik Uzayda Uzaysal Regle Yüzeyler}

Özet: Bu makalede, $\mathbb{H}^{3}$ hiperbolik uzayında uzaysal dönel yüzeyler çalışılmıştır. $\mathbb{H}^{3}$ hiperbolik uzayında bir uzaysal dönel yüzey bir uzaysal doğrunun bir uzaysal eğri boyunca hareket ettirilmesi ile elde edilir. Bu çalışmada, $\mathbb{H}^{3}$ hiperbolik uzayında regle yüzeylerin boğaz noktası, boğaz eğrisi ve dağılma parametresi araştırılmıştır.

Anahtar Kelimeler: Uzaysal Regle Yüzey, Hiperbolik Uzay

\section{INTRODUCTION}

In [1], Turgut and Hacisalihoglu studied timelike ruled surfaces in Minkowski 3-space $\mathbb{R}_{1}^{3}$. They showed that these type surfaces are obtained by moving timelike straight lines along spacelike curves.

In this paper, spacelike ruled surfaces are investigated in Hyperbolic 3-space $\mathbb{H}^{3}$. A ruled surface is a surface obtained by a geodesic $l_{s}^{\alpha}$ moving along a curve $\alpha$. Thus, a ruled surface has a parametrization in $\mathbb{H}^{3}$ as

$\varphi(s, t)=(\cosh t) \alpha(s)+(\sinh t) Z(s)$,

where $\alpha$ is called the base curve and $Z$ the director vector of $l_{s}^{\alpha}$. If the tangent plane is constant along a fixed ruling, then the ruled surface is called a developable surface.

\section{PRELIMINARIES}

Let $R_{1}^{4}$ be 4-dimensional vector space equipped with the scalar product

$\langle x, y\rangle=-x_{1} y_{1}+x_{2} y_{2}+x_{3} y_{3}+x_{4} y_{4}$.

Then, $R_{1}^{4}$ is called Minkowskian or Lorentzian 4space. From now on, the constant angle surface will be proposed in Minkowskian ambient space $R_{1}^{4}$. The Lorentzian norm of $x \in \mathbb{R}_{1}^{4}$ is defined to be

$\|x\|=|\langle x, y\rangle|^{\frac{1}{2}}$

If $\left(x_{0}^{i}, x_{1}^{i}, x_{2}^{i}, x_{3}^{i}\right)$ is the coordinate of $x_{i}$ with respect to canonical basis $\left(e_{0}, e_{1}, e_{2}, e_{3}\right)$ of $R_{1}^{4}$, then the lorentzian cross product of $x_{1}, x_{2}$ and $x_{3}$ is defined by the symbolic determinant as 
$x_{1} \times x_{2} \times x_{3}=\left|\begin{array}{cccc}-e_{0} & e_{1} & e_{2} & e_{3} \\ x_{0}^{1} & x_{1}^{1} & x_{2}^{1} & x_{3}^{1} \\ x_{0}^{2} & x_{1}^{2} & x_{2}^{2} & x_{3}^{2} \\ x_{0}^{3} & x_{1}^{3} & x_{2}^{3} & x_{3}^{3}\end{array}\right|$.

One can easly see that

$\left\langle x_{1} \times x_{2} \times x_{3}, x_{4}\right\rangle=\operatorname{det}\left(x_{1}, x_{2}, x_{3}, x_{4}\right)$.

In [3, 4] and [6] Izumiya at all introduced and investigated differantial geometry of curves and surfaces in $\mathbb{H}^{3}$. The sets

$\left\{x \in R_{1}^{4},\langle x, x\rangle=-1, x_{0} \geq 1\right\}$,

$\left\{x \in R_{1}^{4},\langle x, x\rangle=1\right\}$ and

$\left\{x \in R_{1}^{4},\langle x, x\rangle=0, x_{0} \geq 0\right\}$

are called, respectively, Hyperbolic 3-space $\mathbb{H}^{3}$, de Sitter space $S_{1}^{3}$ and future lightcone at the origin $L C^{*}$. We can give the following background of context in [2].

Let $x: M \rightarrow R_{1}^{4}$ be an immersion of a surface $M$ into $R_{1}^{4}$. We say that $x=\left(x_{0}, x_{1}, x_{2}, x_{3}\right)$ is timelike (resp. spacelike, lightlike) if the induced metric on $M$ via $x$ is Lorentzian (resp. Riemannian, degenerated). If $\langle x, x\rangle=-1$ provided $x_{0}>1$, then $x$ is an immersion of $\mathbb{H}^{3}$.

Since $\mathbb{H}^{3}$ is a Riemannian manifold and regular curve $\gamma$ are reparametrized by arclength, we may assume that $\gamma(s)$ is a unit speed curve. That is, there is a tangent vector $t(s)=\gamma^{\prime}(s)$ with $\|t(s)\|=1$. If $\left\langle t^{\prime}(s), t^{\prime}(s)\right\rangle \neq-1$, then there is a unit vector $n(s)=\frac{t^{\prime}(s)-\gamma(s)}{\left\|t^{\prime}(s)-\gamma(s)\right\|}$

and also $e(s)=\gamma(s) \Lambda t(s) \Lambda n(s)$, where $\Lambda$ denotes the usual vector product. Then we have a pseudo orthonormal frame $\{\gamma(s), t(s), n(s), e(s)\}$ of $R_{1}^{4}$ along $\gamma$.

Taking $\langle t(s), t(s)\rangle \neq-1$, we have the following Frenet-Serre type formulas;

$$
\left\{\begin{array}{l}
\gamma^{\prime}=t(s) \\
t^{\prime}(s)=\kappa_{h}(s) n(s)+\gamma(s) \\
n^{\prime}(s)=-\kappa_{h}(s) t(s)+\tau_{h}(s) e(s) \\
e^{\prime}(s)=-\tau_{h}(s) n(s)
\end{array}\right.
$$

where

$\kappa_{h}(s)=\left\|t^{\prime}(s)-\gamma(s)\right\|$

and

$\tau_{h}(s)=-\frac{\operatorname{det}\left(\gamma(s), \gamma^{\prime}(s), \gamma^{\prime \prime}(s), \gamma^{\prime \prime}(s)\right)}{\left[\kappa_{h}(s)\right]^{2}}$.

Since $\langle t(s), t(s)\rangle \neq-1$, it is easily seen that $\kappa_{h}(s) \neq 0$.

We can show that $\kappa_{h}(s)=0$ if and only if there exists a lightlike vector $c$ such that $\gamma(s)-c$ is a geodesic.

Let $U \subset R^{2}$ be an open subset and $x: U \rightarrow \mathrm{H}^{3}$ be a regular surface. Then, $M=x(U)$ is an embedding of $x$. If $e(u)=\frac{x(u) \Lambda x_{1}(u) \Lambda x_{2}(u)}{\left\|x(u) \Lambda x_{1}(u) \Lambda x_{2}(u)\right\|}$,

then $\langle e, x\rangle=\left\langle e, x_{i}\right\rangle=0$ and $\langle e, e\rangle=1$, where $x_{i}=\frac{\partial x}{\partial u_{i}}$. Thus, de Sitter Gauss image of $x$ is defined by the mapping $E: U \subset R^{2} \rightarrow S_{1}^{3}, E(u)=e(u)$. The lightcone Gauss image of $x$ is also defined by the mapping $L^{ \pm}: U \subset R^{2} \rightarrow L C^{*}, L^{ \pm}(u)=x(u) \pm e(u)$.

Since $d x\left(u_{0}\right)$ and $I_{T p M}$ are identify mappings on the tangent space $T p M$, the derivative $d x\left(u_{0}\right)$ can be identified with $T p M$ relate to identification of $U$ and $M$, that is $d L^{ \pm}\left(u_{0}\right)=I_{T p M} \pm d E\left(u_{0}\right) . \quad$ The linear transformation

$$
S_{p}^{ \pm}:=-d L^{ \pm}\left(u_{0}\right): T p M \rightarrow T p M
$$


is called the hyperbolic shape operator of $M=x(u)$ at $p=x\left(u_{0}\right)$. Also the transformation

$$
A_{p}:=-d E\left(u_{0}\right): T p M \rightarrow T p M
$$

is called the de Sitter shape operator of $M=x(u)$ at $p=x\left(u_{0}\right)$. The eigenvalues of $S_{p}^{ \pm}$and $A_{p}$ are, respectively, denoted by $\overline{K_{i}^{\ddagger}}(p)$ and $K_{i}(p)$, $i=1,2 . \quad \overline{K_{i}^{ \pm}}(p)$ are called the principal curvatures of $M$ in $\mathbb{H}^{3}$ and $S_{p}^{ \pm}$is called the principal curvatures of $M$ in $\mathbb{R}_{1}^{4}$. Since $S_{p}^{ \pm}=-I_{T p M} \pm A_{p}, S_{p}^{ \pm}$and $A_{p}$ have the same eigenvectors and relations

$\overline{K_{i}^{ \pm}}(p)=-1 \pm K_{i}(p)$.

Let $\gamma(s)=x\left(u_{1}(s), u_{2}(s)\right)$ be a unit speed curve on $M=x(u)$ with $p=\gamma\left(s_{0}\right)$. We have the hyperbolic curvature vector $k(s)=t^{\prime}(s)-\gamma(s)$ and the de Sitter normal curvature

$K_{n}^{ \pm}\left(s_{0}\right)=\left\langle t^{\prime}\left(s_{0}\right), L^{ \pm}\left(u_{1}\left(s_{0}\right), u_{2}\left(s_{0}\right)\right)\right\rangle+1$

of $\gamma(s)$ at $p=\gamma\left(s_{0}\right)$. The de Sitter normal curvature depends on the point $p$ and the unit tangent vector of $M$ at $p$ analogous to the Euclidean case. Hyperbolic normal curvature of $\gamma(s)$ is given by

$\overline{K_{n}^{ \pm}}(s)=K_{n}^{ \pm}(s)-1$.

The Hyperbolic Gauss curvature $\overline{K_{h}^{ \pm}}\left(u_{0}\right)$ and the Hyperbolic mean curvature $\overline{H_{h}^{ \pm}}\left(u_{0}\right)$ at $p=x\left(u_{0}\right)$ are given, respectively, by

$\overline{K_{h}^{ \pm}}\left(u_{0}\right)=\operatorname{det} S_{p}^{ \pm}=\overline{K_{1}^{ \pm}}(p) \overline{K_{2}^{ \pm}}(p)$,

$H_{h}^{ \pm}\left(u_{0}\right)=\frac{1}{2} \operatorname{Trace}_{p}^{ \pm}=\frac{\overline{K_{1}^{ \pm}}(p)+\overline{K_{2}^{ \pm}}(p)}{2}$.
The extrinsic (de Sitter) Gauss curvature $K_{e}\left(u_{0}\right)$ and the de Sitter mean curvature $H_{d}\left(u_{0}\right)$ at $p=x\left(u_{0}\right)$ are, respectively, obtained

$K_{e}=\operatorname{det} A p=K_{1}(p) K_{2}(p)$,

$H_{d}\left(u_{0}\right)=\frac{1}{2} \operatorname{TraceAp}=\frac{K_{1}(p)+K_{2}(p)}{2}$.

\section{SPACELIKE RULED SURFACE IN HYPERBOLIC 3-SPACE}

Definition 1 If a geodesic $l_{s}^{\alpha}$ moves along a curve $\alpha$ in Hyperbolic 3 -space $\mathbb{H}^{3}$ we obtain a ruled surface. In this case, geodesic $l_{s}^{\alpha}$ is called the director and $\alpha$ is called the base curve of the ruled surface.

In $\mathbb{H}^{3}$, we will investigate ruled surfaces with spacelike base curves and geodesics.

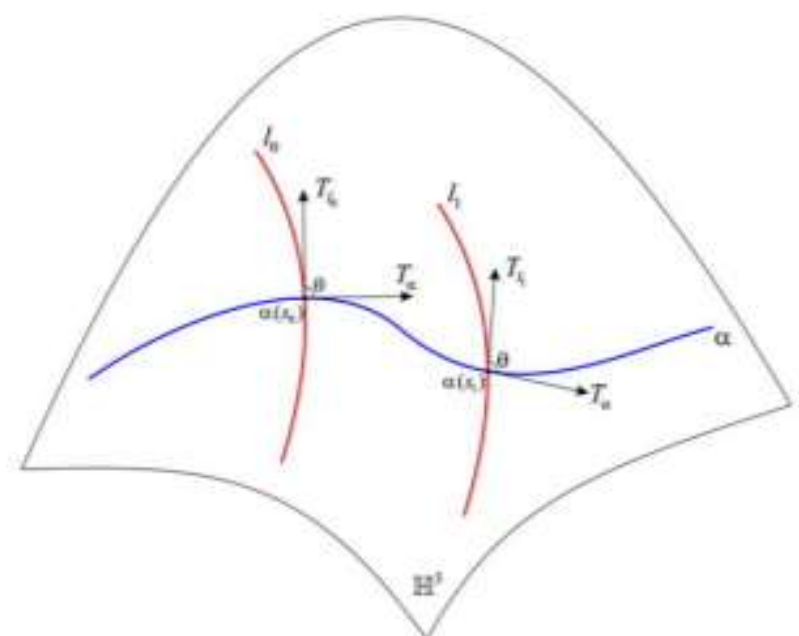

Figure 1. Base curve and direction geodesics of a surface in $\mathbb{H}^{3}$.

Let $\alpha$ be a differentiable unit speed curve in $\mathbb{H}^{3}$ defined by

$\alpha: I \rightarrow \mathrm{H}^{3}$, $\alpha(s)=\left(\alpha_{1}(s), \alpha_{2}(s), \alpha_{3}(s), \alpha_{4}(s)\right) \quad$ where $\{0\} \subset I \subset \mathbb{R}$. Here $\langle\alpha(s), \alpha(s)\rangle=-1,\left\langle\alpha^{\prime}(s), \alpha^{\prime}(s)\right\rangle=\left\langle T_{\alpha}, T_{\alpha}\right\rangle=1$ 
We suppose that

$Z: I \rightarrow S_{1}^{3}, Z(s)=\left(z_{1}(s), z_{2}(s), z_{3}(s), z_{4}(s)\right)$

$\langle Z(s), Z(s)\rangle=1$ and

$\langle\alpha(s), Z(s)\rangle=0, \forall s \in I$.

Let us choose a geodesic in $\mathbb{H}^{3}$ as

$l_{s}^{\alpha}: R \rightarrow \mathrm{H}^{3}, l_{s}^{\alpha}(t)=(\cosh t) \alpha(s)+(\sinh t) Z(s)$ where $\alpha(s)$ is an initial point and $Z(s)$ is the direction vector of $l_{s}^{\alpha}$. Here Frenet components of base curve $\alpha(s)$ are $\left\{T_{\alpha}, N_{\alpha}, B_{\alpha}, \kappa_{h}, \tau_{h}\right\}$. Let $T_{l}$ be tangent of geodesic $l_{s}^{\alpha}$ at the point $\alpha(s)$ and assume that $T_{l}$ and $T_{\alpha}$ are linearly independent for all $s \in I$. If $l_{s}^{\alpha}$ is moved along $\alpha$, then we obtain $(I \times R, \varphi)$ parameterized by $\varphi: I \times R \rightarrow \mathrm{H}^{3}$,

$\varphi(s, t)=\left(\begin{array}{l}(\cosh t) \alpha_{1}(s)+(\sinh t) \alpha_{1}(s), \\ (\cosh t) \alpha_{2}(s)+(\sinh t) \alpha_{2}(s) \\ (\cosh t) \alpha_{3}(s)+(\sinh t) \alpha_{3}(s) \\ (\cosh t) \alpha_{4}(s)+(\sinh t) \alpha_{4}(s)\end{array}\right)$

We will symbolize the ruled surface $\varphi(s, t)$ with M.

Now, we will find an orthonormal base of the tangent space $\chi(M)$ along the curve $\alpha$.

Let $\quad T_{l(s)}=(\cosh t) T_{\alpha(s)}+(\sinh t) T_{Z(s)} \quad$ and $\mathcal{P}_{l}=\frac{T_{l}}{\left\|T_{l}\right\|}$ be the unit tangent of $l_{s}^{\alpha}$. In this case, if we take

$Y=F_{l}^{0}-\left\langle F_{l}^{0}, T_{\alpha}\right\rangle T_{\alpha}$ as a spacelike vector field and its unit as $X=\frac{Y}{\|Y\|}$, then we have

$\|X\|=1$ and $\left\langle X, T_{\alpha}\right\rangle=0,\left\langle T_{\alpha}, T_{\alpha}\right\rangle=1$.

Hence, $\left\{X, T_{\alpha}\right\}$ is an orthonormal base of $\chi(M)$ and we have $\xi=\varphi \wedge X \wedge T_{\alpha}$

called the normal of the ruled surface $M$ in $\mathbb{H}^{3}$, that is

$\chi\left(\mathrm{H}^{3}\right)=S_{p}\left\{X, T_{\alpha}\right\} \oplus S_{p}\{\xi\}$

and

$R_{1}^{4}=S_{p}\left\{X, T_{\alpha}\right\} \oplus S_{p}\{\xi, \varphi\}$.

We symbolize Levi-Civita connections of $R_{1}^{4}$, $\mathbb{H}^{3}$ and $M$, respectively, with $\overline{\bar{D}}, \bar{D}$ and $D$. From Gauss formula, we can write

$\overline{\bar{D}}_{X} Y=\bar{D}_{X} Y+\langle X, Y\rangle \alpha$

$\AA \phi X)=\overline{\bar{D}}_{X} \alpha=I(X)$

and

$\bar{D}_{X} Y=D_{X} Y+\langle A(X), Y\rangle \xi, A(X)=\bar{D}_{X} \xi$.

Taking derivative of the orthonormal frame $\{T, X, \xi\}$ along the curve $\alpha$, we obtain

$\left[\begin{array}{c}\bar{D}_{T} T \\ \bar{D}_{T} X \\ \bar{D}_{T} \xi\end{array}\right]=\left[\begin{array}{ccc}0 & a & b \\ -a & 0 & c \\ b & c & 0\end{array}\right]\left[\begin{array}{c}T \\ X \\ \xi\end{array}\right]$

where

$a=\left\langle\bar{D}_{T} T, X\right\rangle, b=\left\langle\bar{D}_{T} T, \xi\right\rangle, c=\left\langle\bar{D}_{T} X, \xi\right\rangle$.

For the system $\{\alpha, T, X, \xi\}$ along $\alpha$, using Gauss formula we obtain

$\left[\begin{array}{c}\overline{\bar{D}}_{T} \alpha \\ \overline{\bar{D}}_{T} T \\ \overline{\bar{D}}_{T} X \\ \overline{\bar{D}}_{T} \xi\end{array}\right]=\left[\begin{array}{cccc}0 & 1 & 0 & 0 \\ -1 & 0 & a & b \\ 0 & -a & 0 & c \\ 0 & -b & -c & 0\end{array}\right]\left[\begin{array}{c}\alpha \\ T \\ X \\ \xi\end{array}\right]$

which can be given also as 


$$
\left\{\begin{array}{l}
\overline{\bar{D}}_{T} \alpha=T \\
\overline{\bar{D}}_{T} T=-\alpha+a X+b \xi \\
\overline{\bar{D}}_{T} X=-a T+c \xi \\
\overline{\bar{D}}_{T} \xi=-b T-c X
\end{array}\right.
$$

For the ruled surface $M$ given by the parametrization $\varphi: I \times R \rightarrow \mathrm{H}^{3}$,

$\varphi(s, t)=(\cosh t) \alpha(s)+(\sinh t) X(s)$

we have

$$
\left\{\begin{array}{l}
E=\left\langle\varphi_{s}, \varphi_{s}\right\rangle=(\cosh t-a \sinh t)^{2}+c^{2} \sinh ^{2} t \\
F=\left\langle\varphi_{s}, \varphi_{t}\right\rangle=0 \\
G=\left\langle\varphi_{t}, \varphi_{t}\right\rangle=1
\end{array}\right.
$$

Since $E G-F^{2}>0$, the ruled surface $M$ is spacelike in $\mathbb{H}^{3}$. Let us denote domain of $t$ by $J$ - $\varphi_{t_{0}}: I \times t_{0} \rightarrow M$,

$\varphi_{t_{0}}\left(s, t_{0}\right)=\left(\cosh t_{0}\right) \alpha(s)+\left(\sinh t_{0}\right) X(s)$

determines a curve of $M$, where $t=t_{0}$ is constant. The tangent vector field of this curve is $A=\left(\cosh t_{0}-a \sinh t_{0}\right) T(s)+\left(c \sinh t_{0}\right) \xi(s)(3.10)$.

Since $M$ is spacelike, $\langle A, A\rangle>0$ and $\varphi_{t_{0}}$ is a spacelike curve. Also,

$\langle X, A\rangle=0$.

\section{DEVELOPABLE RULED SURFACE IN HYPERBOLIC 3- SPACE $\mathbb{H}^{3}$}

Definition 2 If the tangent planes of a ruled surface in $\mathbb{H}^{3}$ are the same along its main geodesics, then this ruled surface is called a developable ruled surface.

Theorem 1 Let $M$ be a spacelike ruled surface in $\mathbb{H}^{3}$. Then, the tangent planes are the same along a main geodesic if and only if $c=0$.

Proof Let $M$ be a spacelike ruled surface, and suppose that the tangent planes are the same along one of its main geodesics. We consider the tangent vector field

$$
A=\left(\cosh t_{0}-a \sinh t_{0}\right) T(s)+\left(c \sinh t_{0}\right) \xi(s)
$$

of the curve $\varphi_{t_{0}}: I \times\left\{t_{0}\right\} \rightarrow M$ that passed through $t_{0} \in I$. Since $\varphi_{t_{0}}$ is parameter curve of $M$, the vector $A$ is in the tangent plane of the surface $M$. Thus, $c=0$.

Conversely, assume that $c=0$. In this case, since

$$
A=\left(\cosh t_{0}-a \sinh t_{0}\right) T(s)
$$

and

$T_{\varphi\left(t_{0}, s\right)} M=S p\{T, X\}=S p\{T, A\}$,

the tangent planes are the same along one of its main geodesics.

Corollary 1 The spacelike ruled surface $M$ in $\mathbb{H}^{3}$ is developable if and only if $c=0$.

Corollary 2 For a spacelike ruled surface $M$ in $\mathbb{H}^{3}$, we get

$$
\begin{aligned}
& b=-\operatorname{det}\left(T, X, \alpha, \overline{\bar{D}}_{T} T\right) \\
& c=-\operatorname{det}\left(T, X, \alpha, \overline{\bar{D}}_{T} X\right)
\end{aligned}
$$

Remark 1 Since stereographic projection is conformal mapping, using stereographic projection, spacelike ruled surfaces in Minkowskian model of $\mathbb{H}^{3}$ is visualized in Poincare ball model of $\mathbb{H}^{3}$.

Example 1 Let us take a ruled surface $M$ in $\mathbb{H}^{3}$ given by the parametrization $\varphi: I \times R \rightarrow \mathrm{H}^{3}$,

$$
\varphi(s, t)=(\cosh t) \alpha(s)+(\sinh t) X(s) .
$$

If

$\alpha(s)=(\cosh s, \sinh s \cos , \sinh s \sin s, 0)$

and

$X(s)=(\cosh s, \sqrt{2} \cos s, \sqrt{2} \sin s, \sinh s)$

are choosen, then $\varphi(s, t)$ is a spacelike ruled surface in $\mathbb{H}^{3}$. The base curve $\alpha$ is also spacelike and 
$\langle\alpha(s), \alpha(s)\rangle=-1$.

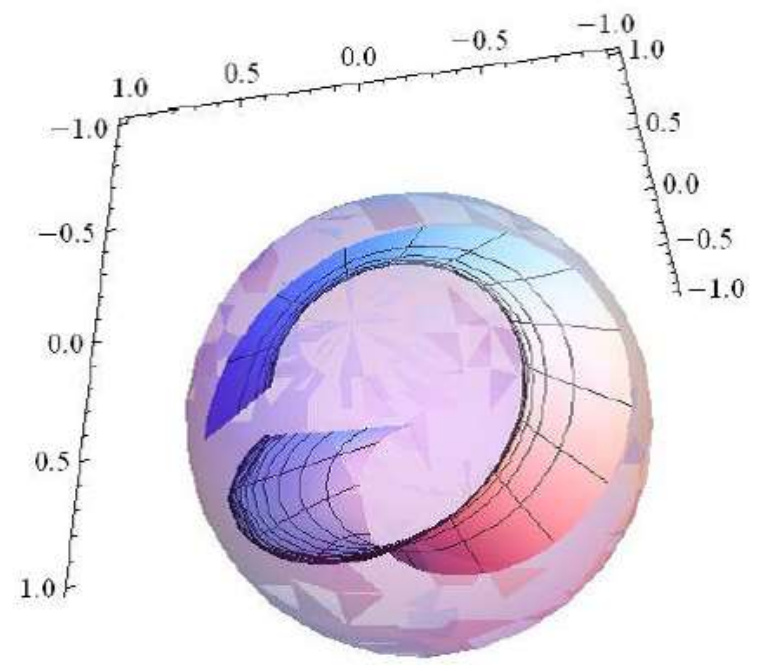

Figure 2. Ruled surface in Hyperbolic 3-space $\mathbb{H}^{3}$.

\subsection{Position Vector of a Central Point in Hyperbolic 3-Space $\mathbb{H}^{3}$}

Definition 3 Let an undevelopable ruled surface be given in $\mathbb{H}^{3}$. If there exists a comman perpendicular of two neighbor main geodesics of the ruled surface, then the foot of this perpendicular on principal geodesic is called the striction or central point.

Definition 4 When the straight line of an undevelopable ruled surface in $\mathbb{H}^{3}$ creates the ruled surface through the base curve, then the geometrical place of the central points of the ruled surface is called the striction curve of $M$.

If $w$ is the distance between the central point and the base curve of the undevelopable ruled surface, then the position vector $\bar{\alpha}(s)$ can be defined by

$$
\bar{\alpha}(s, w)=(\cosh w) \alpha(s)+(\sinh w) X(s),(4.1)
$$

where $\alpha(s)$ is the position vector of the base curve and $X(s)$ is the direction vector of the main geodesic.

The parameter $w$ can be written as the combination of the position vector of the base curve and the direction vector of the ruled surface. Let first two of three neighbor geodesic of spacelike ruled surface be $l_{s}^{\alpha}=(\cosh t) \alpha(s)+(\sinh t) X(s)$

and

$l_{s+\Delta s}^{\alpha}=(\cosh t) \alpha(s+\Delta s)+(\sinh t) X(s+\Delta s)$,

where $X(s)$ and $X(s)+\bar{D}_{T(s)} X(s)$ are the direction vectors of these main geodesics, respectively. Also let $P, P^{\prime}$ and $Q, Q^{\prime}$ be the feet on the main geodesics of the common perpendicular of the neighbour geodesic. Thus, $P$ and $Q$ are two different central points. The direction of the common perpendicular first two main geodesics are linearly dependent to the vector

$\alpha(s) \wedge X(s) \wedge\left[X(s)+\bar{D}_{T(s)} X(s)\right]$.

Therefore,

$$
\begin{gathered}
\alpha(s) \wedge X(s) \wedge\left[X(s)+\bar{D}_{T(s)} X(s)\right]= \\
=\alpha(s) \wedge X(s) \wedge \bar{D}_{T(s)} X(s)
\end{gathered}
$$

The vector $\stackrel{\text { man }}{P Q}$ coincides with the vector $\stackrel{\max }{P P}$ 'in the limiting position, and $P Q$ will be the tangent vector of the striction curve. Since

$\left\{\begin{array}{l}\langle X(s), P Q\rangle=0 \\ \left\langle X(s)+\bar{D}_{T(s)} X(s), P Q\right\rangle=0\end{array}\right.$,

we obtain

$\left\langle\bar{D}_{T(s)} X(s), P Q\right\rangle=0$.

Thus,

$\left\langle\bar{D}_{T(s)} X(s), \bar{D}_{T(s)} \bar{\alpha}(s)\right\rangle=0$.

On the other hand, since

$\bar{D}_{T(s)} \bar{\alpha}(s)=\overline{\bar{D}}_{T(s)} \bar{\alpha}(s)-\langle T(s), \bar{\alpha}(s)\rangle \bar{\alpha}(s)$,

we obtain 
$\bar{D}_{T(s)} \bar{\alpha}(s)=\overline{\bar{D}}_{T(s)} \bar{\alpha}(s)$.

Consequently,

$\left\langle\overline{\bar{D}}_{T(s)} X(s), \overline{\bar{D}}_{T(s)} \bar{\alpha}(s)\right\rangle=0$

and then

$\frac{\sinh w}{\cosh w}=\frac{a}{a^{2}+c^{2}}$ or $w=\arctan h\left(\frac{a}{a^{2}+c^{2}}\right)$

So, the position vector of the striction curve is

$\bar{\alpha}(s, w)=(\cosh w) \alpha(s)+\frac{a}{a^{2}+c^{2}}(\cosh w) X(s)$

Corollary 3 The distance between the central point of the undevelopable ruled surface and the base curve is constant.

Proof Since

$\langle X(s), P Q\rangle=0$

we obtain

$\left\langle X(s), \bar{D}_{T(s)} \bar{\alpha}(s)\right\rangle=0$ and

$\overline{\bar{D}}_{T(s)} \bar{\alpha}(s)=\bar{D}_{T(s)} \bar{\alpha}(s)$.

Thus, $\cosh w \frac{d w}{d s}=0$ and $\frac{d w}{d s}=0$. So, $w$ is constant.

Theorem 2 Striction curve of a spacelike ruled surface in $\mathbb{H}^{3}$, which is undevelopable, is independent of choosing the base curve.

Proof Let us denote two spacelike ruled surfaces in $H^{3}$ by

$\varphi(t, v)=(\cosh v) \alpha(t)+(\sinh v) X(t)$

$\varphi(t, v)=(\cosh v) \beta(t)+(\sinh v) X(t)$

where $\alpha$ and $\beta$ are two different base curves of the spacelike ruled surface. Then, the striction curves of the spacelike ruled surfaces are $\bar{\alpha}(t)=(\cosh v) \alpha(t)+\frac{a}{a^{2}+c^{2}}(\cosh v) X(t)$

$\bar{\beta}(t)=(\cosh v) \beta(t)+\frac{a}{a^{2}+c^{2}}(\cosh v) X(t)$

If we subtract $\bar{\beta}(t)$ from $\bar{\alpha}(t)$ and use (4.1), we obtain

$\bar{\alpha}(t)-\bar{\beta}(t)=0$

That completes the proof.

Theorem 3 Let $M$ be an undevelopable spacelike ruled surface. Then, the point $\varphi\left(s, v_{0}\right)$ is a striction point on the straight line, passing through the point $\alpha(s)$, if and only if $\bar{D}_{T} X$ is a normal vector of the tangent plane on the point $\varphi\left(s, v_{0}\right)$.

Proof Suppose that $\bar{D}_{T} X$ is a normal vector of the tangent plane on the point $\varphi\left(s, v_{0}\right)$. Since tangent vector field of the curve $\varphi_{v_{0}}: I \times\left\{v_{0}\right\} \rightarrow M$ is

$A=\left(\cosh v_{0}-b \sinh v_{0}\right) T(s)+\left(c \sinh v_{0}\right) \xi(s)$

then

$\left\langle\bar{D}_{T(s)} X(s), A\right\rangle=0$.

Thus, we obtain

$-a \cosh v_{0}+a^{2} \sinh v_{0}+c^{2} \sinh v_{0}=0$

and

$\frac{\sinh v_{0}}{\cosh v_{0}}=\frac{a}{a^{2}+c^{2}}$.

Therefore, $\varphi\left(s, v_{0}\right)$ is a central point of $M$.

Conversely, suppose that $\varphi\left(s, v_{0}\right)$ is a central point with geodesic, passing through the points $\alpha(s)$. Thus,

$\left\langle\bar{D}_{T} X, X\right\rangle=0$, 
$\left\langle\bar{D}_{T} X, A\right\rangle=-a\left(\cosh v_{0}-a \sinh v_{0}\right)+c^{2} \sinh v_{0}$

Since $\varphi\left(s, v_{0}\right)$ is a central point, then we get

$-a\left(\cosh v_{0}-a \sinh v_{0}\right)+c^{2} \sinh v_{0}=0$.

Hence, we obtain

$\left\langle\bar{D}_{T} X, A\right\rangle=0$.

So, $\bar{D}_{T} X$ is a normal vector of the tangent plane at the point $\varphi\left(s, v_{0}\right)$.

Remark 1 Let $\bar{D}_{T} X$ be a normal vector of the tangent plane on the striction point. Since

$\left\langle\bar{D}_{T(s)} X(s), \bar{D}_{T(s)} X(s)\right\rangle=a^{2}+c^{2}>0$,

$\bar{D}_{T} X$ is a spacelike normal vector field.

Theorem 4 Let $M$ be an undevelopable spacelike ruled surface. Then, the striction curve

$\bar{\alpha}(s)=(\cosh w) \alpha(s)+\frac{a}{a^{2}+c^{2}}(\cosh w) X(s)$

is spacelike.

Proof We need to show that the tangent vector field of the striction curve $\bar{\alpha}$ is spacelike. It is clear that

$$
\left\langle\overline{\bar{D}}_{T(s)} \bar{\alpha}(s), \overline{\bar{D}}_{T(s)} \bar{\alpha}(s)\right\rangle=\frac{c^{2}}{a^{2}+c^{2}} \cosh ^{2} w>0
$$

where

$$
\overline{\bar{D}}_{T(s)} \bar{\alpha}(s)=
$$

$=(\cosh w) \overline{\bar{D}}_{T(s)} \alpha(s)+\frac{a}{a^{2}+c^{2}}(\cosh w) \overline{\bar{D}}_{T(s)} X(s) \lambda=(\cosh t) \frac{\operatorname{det}\left(\alpha(s), X(s), T(s), \bar{D}_{T(s)} X(s)\right)}{\left\langle\bar{D}_{T(s)} X(s), \bar{D}_{T(s)} X(s)\right\rangle}$

Since $\left\langle\overline{\bar{D}}_{T(s)} \bar{\alpha}(s), \overline{\bar{D}}_{T(s)} \bar{\alpha}(s)\right\rangle>0, \quad \bar{\alpha}(s)$ is a spacelike curve.

\subsection{Dispersion Parameter of Ruled Surface in Hyperbolic 3-Space $\mathbb{H}^{3}$}

Let the base curve of a spacelike ruled surface $M$ be the striction curve. Then, the distance from the striction point to the base curve is

$$
w=\arctan h\left(\frac{a}{a^{2}+c^{2}}\right)=0 .
$$

Hence, we have

$a=0$

and since

$\bar{D}_{T(s)} X(s)=-a T(s)+c \xi(s)$,

the vector field $\bar{D}_{T(s)} X(s)$ and the normal of surface $\xi(s)$ are linearly independent. Therefore, there exists $\lambda \in R$ for the equality $\xi(s)=\lambda \bar{D}_{T(s)} X(s)$.

On the other hand, since

$\xi(s)=\lambda \bar{D}_{T(s)} X(s)$

and

$\varphi=(\cosh t) \alpha(s)+(\sinh t) X(s)$,

we have

$\xi(s)=(\cosh t)[\alpha(s) \wedge X(s) \wedge T(s)]$.

Therefore, we have

$\lambda \bar{D}_{T(s)} X(s)=(\cosh t)[\alpha(s) \wedge X(s) \wedge T(s)]$.

If we take scalar product with $\bar{D}_{T(s)} X(s)$ of both sides of the above equality, then we have

where $\lambda$ is called the dispertion parameter of the spacelike ruled surface in $\mathbb{H}^{3}$. 
Since the vector field $\bar{D}_{T(s)} X(s)$ and the normal of the surface are linearly independent, $\bar{D}_{T(s)} X(s)$ is a spacelike vector field.

Theorem 5 The spacelike ruled surface $M$ is developable if and only if the dispersion parameter of $M$ is zero.

Proof From Theorem 2 and Corollary 1, we get

$$
c=-\operatorname{det}\left(T, X, \alpha, \overline{\bar{D}}_{T} X\right)=0
$$

and so it is clear from the definition of the dispersion parameter that

$$
\lambda=(\cosh t) \frac{\operatorname{det}\left(\alpha, T, X, \bar{D}_{T} X\right)}{\left\langle\bar{D}_{T} X, \bar{D}_{T} X\right\rangle}=0 .
$$

Definition 5 If there exists a curve that cuts vertically each geodesics of the ruled surface in $\mathbb{H}^{3}$, then this curve is called an orthogonal trajectory of the ruled surface.

Theorem 6 Let $M$ be a spcelike ruled surface in $\mathbb{H}^{3}$. Then, there is only one orthogonal trajectory passing through every point of $M$.

Proof Let $M$ be a spacelike ruled surface given by the parametrization $\varphi: I \times J \rightarrow \mathrm{H}^{3} \subset R_{1}^{4}$,

$\varphi(s, v)=(\cosh v) \alpha(s)+(\sinh v) Z(s)$.

Then, the orthogonal trajectory of $M$ is $\beta: P / M, P / \subset I$

$$
\beta(t)=(\cosh f(t)) \alpha(t)+(\sinh f(t)) Z(t) .
$$

Since

$$
\left\langle\bar{D}_{T(t)} \beta(t), Z(t)\right\rangle=0,
$$

we get

$$
f(t)=-\int\left\langle\alpha^{\prime}(t), Z(t)\right\rangle d t+h,
$$

where $\langle Z(t), Z(t)\rangle=1$.

If we take

$$
F(t)=-\int\left\langle\alpha^{\prime}(t), Z(t)\right\rangle d t,
$$

we get

$$
f(t)=F(t)+h .
$$

Since $h$ is choosen arbitrary, there are a lot of curves that satisfy the condition

$$
\left\langle\bar{D}_{T(t)} \beta(t), Z(t)\right\rangle=0 \text {. }
$$

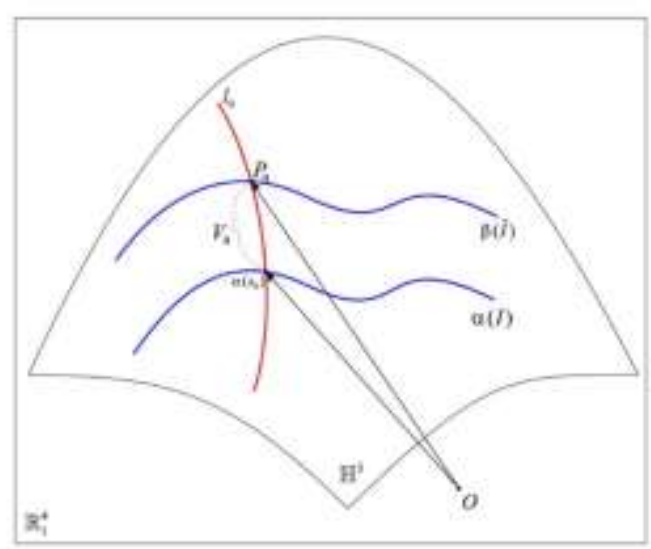

Figure 3. Orthogonal trajectory of a ruled surface.

Let us now find $s \in R$ such that

$$
p_{0}=\cosh (F(t)+h) \alpha(t)+\sinh (F(t)+h) Z(t)
$$

Since

$$
p_{0}=\cosh \left(v_{0}\right) \alpha\left(t_{0}\right)+\sinh v_{0} Z\left(t_{0}\right),
$$

we get

$$
\begin{aligned}
& \cosh (f(t)) \alpha(t)+\sinh (f(t)) Z(t)= \\
& =\left(\cosh v_{0}\right) \alpha\left(t_{0}\right)+\sinh \left(v_{0}\right) Z\left(t_{0}\right)
\end{aligned}
$$

So, $\alpha\left(t_{0}\right)=\alpha(t), v_{0}=f(t)$. If we choose interval $I$ such that $\alpha$ is one to one, then we get $t=t_{0}$. Thus, $h=f\left(t_{0}\right)-F\left(t_{0}\right)$. Consequently, there exists only one orthogonal trajectory passing through the point $P_{0}$. Therefore, ${ }^{/} /$must be equal to $I$.

Theorem 7 Let $M$ be an undevelopable spcelike ruled surface in $\mathbb{H}^{3}$. Then, the longest distance along the orthogonal trajectors between any two 
geodesics of $M$ is the distance measured along the curve $\varphi_{t}: I \rightarrow M$

corresponding to

$t=\frac{1}{2} \arctan h\left(\frac{2 a}{1+a^{2}+c^{2}}\right)$.

Proof Let us take two geodesic passing through the points $\alpha\left(s_{1}\right)$ and $\alpha\left(s_{2}\right)$, where $s_{1}, s_{2} \in I$ and $s_{1}<s_{2}$. Also, let us denote the distance obtained along the orthogonal trajector $s=$ constant between these geodesics by $d(t)$. Then,

$$
\begin{aligned}
& d(t)=\int_{s_{1}}^{s_{2}}\|A\| d s \\
& =\sqrt{(\cosh t-a \sinh t)^{2}+c^{2} \sinh ^{2} t}\left(s_{2}-s_{1}\right)
\end{aligned}
$$

where

$$
A=(\cosh t-a \sinh t) T(s)-(c \sinh t) \xi(s) .
$$

If $d^{\prime}(t)=0$, then $d(t)$ takes the maximum value. Hence, we get

$$
t=\frac{1}{2} \arctan h\left(\frac{2 a}{1+a^{2}+c^{2}}\right) .
$$

Theorem 8 Let $M$ be a spacelike ruled surface in $\mathbb{H}^{3}$ The geodesics of $M$ are both asymptotic and geodesic curves.

Proof Let $X$ be the tangent vector field of a geodesic of a spacelike ruled surface $M$. Since every geodesic in ruled surface $M$, it is a geodesic in $\mathbb{H}^{3}$. Thus, we get $\bar{D}_{X} X=0$. From Gauss equation, we also get

$$
\bar{D}_{X} X=D_{X} X-\left\langle S^{ \pm}(X), X\right\rangle \xi .
$$

Thus,

$D_{X} X=\left\langle S^{ \pm}(X), X\right\rangle \xi$.

Therefore,

$$
D_{X} X \in \chi(M)
$$

and $\left\langle S^{ \pm}(X), X\right\rangle \xi \in \chi^{\perp}(M)$. Since the metric on $M$ is nondegenered, we get

$\chi\left(\mathrm{H}^{3}\right)=\chi(M) \oplus \chi^{\perp}(M)$

and

$\chi(M) \cap \chi^{\perp}(M)=\{0\}$.

Thus, $D_{X} X=0$ and $\left\langle S^{ \pm}(X), X\right\rangle=0$.

Theorem 9 Let $M$ be a spacelike ruled surface in $\mathbb{H}^{3}$. Then, $\bar{K}_{h}^{ \pm}(p) \leq 0$ for all $p \in M$, where $\bar{K}_{h}^{ \pm}$ is the Gaussian curvature function of $M$ in $\mathbb{H}^{3}$.

Proof Let $X$ be the tangent vector field of the main geodesic at the point $p \in M$. And take the orthonormal basis $\{X, Y\}$ of $\chi(M)$. Since $M$ is a ruled surface, $X$ and $Y$ are spacelike vector fields. The Weingarten operator $S^{ \pm}$of $M$ can be written

$$
\begin{aligned}
& S^{ \pm}(X)=\left\langle S^{ \pm}(X), X\right\rangle X+\left\langle S^{ \pm}(X), Y\right\rangle Y \\
& S^{ \pm}(Y)=\left\langle S^{ \pm}(Y), X\right\rangle X+\left\langle S^{ \pm}(Y), Y\right\rangle Y
\end{aligned}
$$

In this case, the matrix

$$
S^{ \pm}=\left[\begin{array}{cc}
\left\langle S^{ \pm}(X), X\right\rangle & \left\langle S^{ \pm}(X), Y\right\rangle \\
\left\langle S^{ \pm}(Y), X\right\rangle & \left\langle S^{ \pm}(Y), Y\right\rangle
\end{array}\right]
$$

is corresponding to Weingarten operator $S^{ \pm}$. On the other hand, the Weingarten operator $S^{ \pm}$is self-adjoint,

$$
\left\langle S^{ \pm}(X), Y\right\rangle=\left\langle S^{ \pm}(Y), Y\right\rangle .
$$

Also, from Theorem 8, we get

$$
\left\langle S^{ \pm}(X), X\right\rangle=0,\left\langle S^{ \pm}(Y), Y\right\rangle=0 .
$$

Hence,

$$
\bar{K}_{h}^{ \pm}=\operatorname{det} S^{ \pm}=-\left\langle S^{ \pm}(X), Y\right\rangle^{2} .
$$

Theorem 10 Let $M$ be a spacelike ruled surface in $\mathbb{H}^{3}$. Then, 


$$
\begin{aligned}
& \alpha \wedge T \wedge X=\xi \\
& T \wedge X \wedge \xi=-\alpha \\
& X \wedge \xi \wedge \alpha=T \\
& \xi \wedge \alpha \wedge T=-X
\end{aligned}
$$

where $T$ is the unit tangent vector of the base curve, $\alpha$ is the position vector both of the base curve of $M, X$ is the unit tangent vector field of the main geodesic of $M$, and $\xi$ is the unit normal vector field of $M$.

\section{REFERENCES}

[1]. Turgut,A.,Hacisalihoğlu,H. Timelike ruled surface in the Minkowski 3-space, Far East J.Math.Sci.5 (1997) 83-90.

[2]. Turgut,A. Spacelike and Timelike Ruled Surface on the Minkowski 3-Space, Ph. D. Thesis, Ankara University,1995.
[3]. Izumıya, S.; Saj1, K.; Takahashı, M. Horospherical flat surfaces in Hyperbolic 3space. J.Math.Soc.Japan. 87 (2010) 789-849.

[4]. Izumıya, S.; Pe1, D.; Fuster, M. The horospherical geometry of surfaces in hyperbolic 4-spaces. Israel Journal of Mathematics. 154 (2006) 361-379.

[5]. Sabuncuoğlu,A. Generelized Ruled Surface, Associate Proffesorshiph Thesis, Ankara University, 1982.

[6]. Thas, C. A gauss map on hypersurfaces of submanifolds in Euclidean spaces. J.Korean Math. Soc. 16 (1979) 17-27.

[7]. O’Neill,B. Semi-Riemannian Geometry with Applications to Relativity, Academic Press, New York, 1983.

[8]. Fencel,W. Elementary Geometry in Hyperbolic Space, Walter de Gruyter, New York, 1989.

[9]. Ratcliffe J. Foundations of Hyperbolic Manifolds, Springer,2000. 